\title{
Combining damage and friction to model compressive damage growth in fibre-reinforced composites
}

Journal of Composite Materials $0(0) \mathrm{I}-13$

(C) The Author(s) 2014 Reprints and permissions: sagepub.co.uk/journalsPermissions.nav DOI: 10.1 I77/00219983|45496/4 jcm.sagepub.com

@SAGE

\author{
Renaud Gutkin' and Silvestre T Pinho ${ }^{2}$
}

\begin{abstract}
A material model for unidirectional fibre-reinforced composites coupling damage to the friction acting on newly created microcracks is developed. While existing material models accounting for progressive damage assume that microcracks remain traction free under compressive load, the present model accounts for contact and friction at microcrack closure. The model is validated against experimental data and it is shown that friction can account for part of the non-linear response and the hysteresis loops typically observed in the shear response of composites. Further validation against simple crushing tests is performed and shows that the physics behind crushing is well captured.
\end{abstract}

\section{Keywords}

Crushing, damage mechanics, friction

\section{Introduction}

Modelling compressive damage growth is of great interest in the application of many composites. For instance, being able to predict the energy absorption of crash structures is a key enabler for the introduction of composites in automotive applications. Compressive damage growth also plays an important role in predicting the response of bolted joints where bearing failure is involved or in impact analyses where extensive compressive damage is created at the contact between the impactor and structure.

Figure 1 shows typical failure modes observed in longitudinal (a and b) and transverse (c) compressive failure of unidirectional composites. In these three cases, failure localises on a fracture plane oriented at $30^{\circ}, 45^{\circ}$ and $53^{\circ}$, respectively, to the loading direction. Failure is predominantly initiated and driven by the shear component of the traction vector acting on that plane (resolved from the applied compressive loading), as shown in Figure 1(d) to (f). Pinho et al. ${ }^{1}$ developed a progressive damage model in which the response of the material is obtained taking into account the intended traction response on the fracture plane. For the cases in Figure 1(a) and (c), this means that the material response depends on the response of the shear traction, which in turn is assumed to follow a linear softening law. This concept is very practical as it enables the description of the material directly from the damage and not by assuming the effect of the damage on the elastic constants as for example done in Gutkin and Pinho $^{2}$ and Maimi et al. ${ }^{3}$ This concept is close to the fixed crack model originally developed for concrete (see Jirásek and Patzák ${ }^{4}$ for a review) and which makes it possible to define the response of the material based on a constitutive law defined on a fracture plane, for which the orientation remains fixed during the loading history.

Using a linear softening traction separation law to describe progressive damage, it is possible to predict accurately failure of complex structures. ${ }^{1-3,5}$ However, with this method, only the fracture energy spent during growth is included; once damage is fully grown, the microckracks are left traction free. But energy absorption for compressively loaded composite structures is

\footnotetext{
'Swerea SICOMP AB, SE-43I 22, Mölndal, Sweden

${ }^{2}$ The Composites Centre, Department of Aeronautics, South Kensington Campus, Imperial College London, London, UK
}

Corresponding author:

Renaud Gutkin, Swerea SICOMP AB, SE-43I 22 Mölndal, Sweden.

Email: renaud.gutkin@swerea.se 

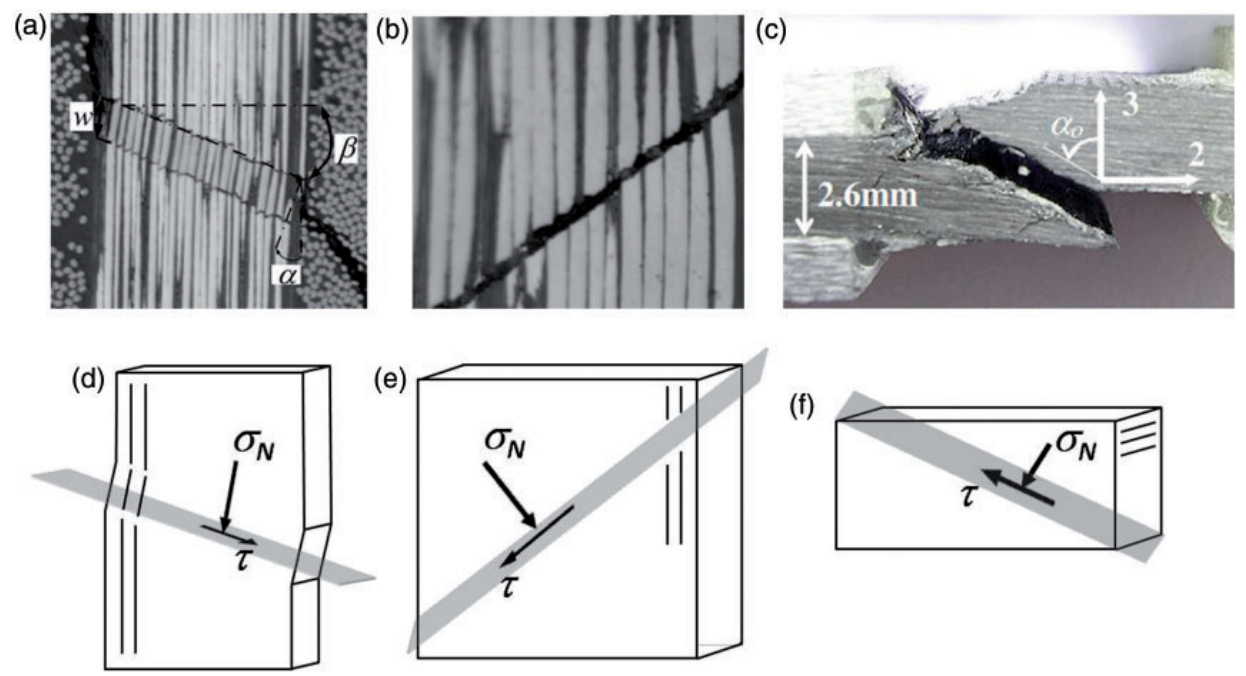

Figure I. Compressive failure in unidirectional composites: (a) fibre kinking, (b) fibre shear failure and (c) matrix failure (from Pinho et al. $\left.{ }^{\prime}\right)$. ( $d-f$ ) Corresponding resolved normal and shear components of the traction vector on the fracture plane.

the result of several energy dissipating mechanisms at the material scale: the energy spent to create new damage areas and the friction energy due to the sliding on the newly created crack faces. ${ }^{6}$ The effect of friction in modelling interlaminar crack growth or debonding has been investigated in the work by Tvergaard $^{7}$ and Chaboche et al. ${ }^{8}$ In these works, damage growth is represented by a traction separation law, defining a parabolic relationship between traction and separation, also referred to as Tvergaard's model. The two models differ in the method used to include the friction. In Tvergaard, ${ }^{7}$ the friction term is introduced once full decohesion has been achieved, while in Chaboche et al. $^{8}$ friction is introduced as soon as decohesion starts. These two models offer two extreme steady state regimes where the development of the frictional term is overlooked. In modelling cracking in concrete, Ragueneau et al. ${ }^{9}$ proposed a continuum formulation to couple damage and friction. From the expression of the state potential, it is shown that it is possible to couple both phenomena with a progressive evolution of the frictional contribution.

The present paper introduces a general model coupling damage and friction in the framework of the fixed crack concept, therefore making it possible to account for local effects, such as friction on microcracks, in a physical sense. The approach developed by Pinho et al., to introduce damage through a fixed crack model, is combined to the physical coupling of damage and friction proposed by Ragueneau et al. ${ }^{9}$ Therefore, these two concepts are first reviewed independently and before the general formulation is presented. The current paper is focused on the application of the model to matrix shear and compressive failure, including a discussion on model parameters identification.

\section{Framework of the model and background}

\section{Framework of the model}

The scope of the present work is limited to damage occurring in compression, i.e. for $\sigma_{\mathrm{N}} \leq 0$ shown in Figure 1(d-f), and on fracture planes with a normal $n$ perpendicular to the fibre direction, as shown in Figure 2(b). Stress states triggering damage that do not fulfill the condition above, e.g. tensile damage and fibre failure in compression, are not the focus of the paper.

\section{Fixed crack model}

As discussed in the introduction, the term 'fixed crack model' is used to describe a material model where the constitutive material response is obtained by a relation between the stress and the strain on a given fracture plane of fixed orientation. The method is schematically described in Figure 2. Before damage initiation, and therefore before a fracture plane is created, the response at the ply level follows a linear elastic and transversely isotropic behaviour, which is illustrated in Figure $2 \mathrm{a}$ with the stiffness matrix $\boldsymbol{C}$. Once the damage initiation is predicted and a fracture plane identified, see Figure 2(b), the strain $\varepsilon$ is rotated onto coordinates aligned with the fracture plane and is used to calculate the traction vector on the fracture plane, given a constitutive law which accounts for, e.g., damage and friction. This new inelastic traction is then incorporated into the 

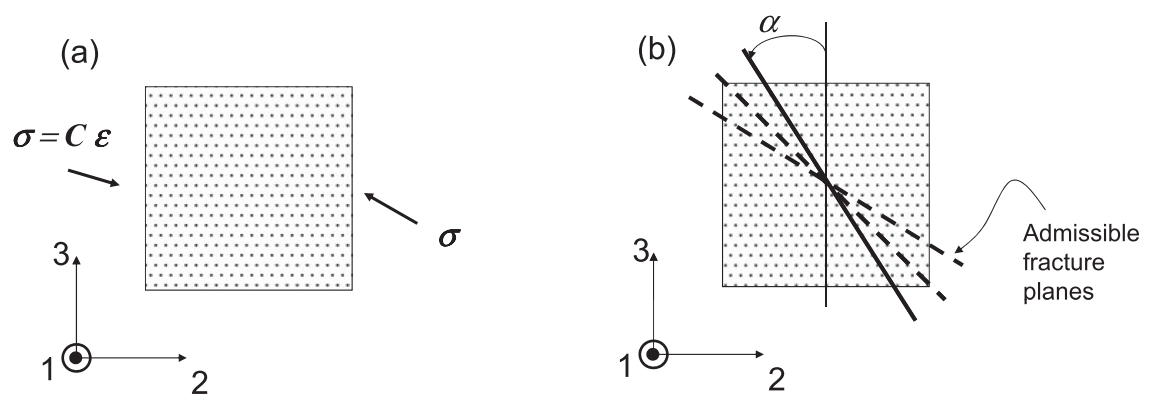

(c)

$(T: \varepsilon) \cdot \boldsymbol{n}$

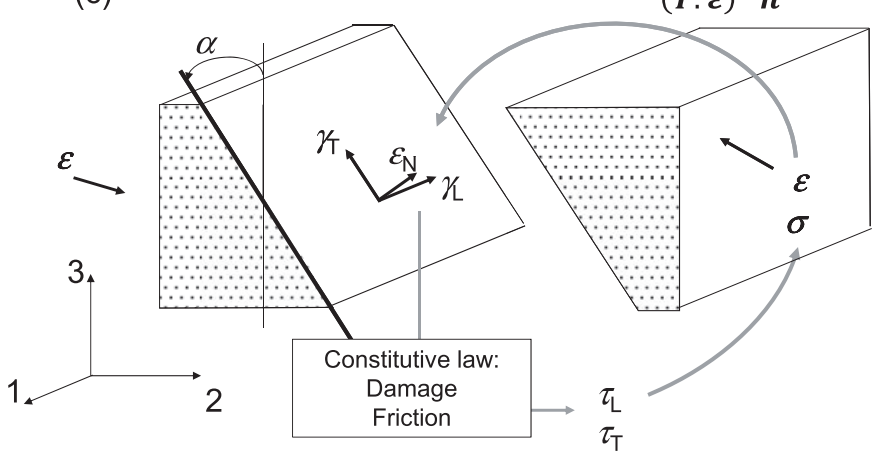

Figure 2. Description of the fixed crack method: (a) initial elastic response, (b) admissible fracture planes for matrix cracking and (c) definition of traction vector acting on a general fracture plane and calculation process.

stress tensor and resolved back to the material coordinates, as shown in Figure 2(c). The hypothesis of strain equivalence introduced by Lemaitre and Chaboche ${ }^{10}$ is assumed here; that is to say that the strain in the damaged state under the new stress is equivalent to the strain in the undamaged state under the initial elastic stress.

It is possible to allow the angle of the fracture plane to rotate during damage growth, rotating crack model; however, this would be costly numerically and is not investigated in the present work. Using the fixed crack approach, it is possible to account in a simple way for the observation made earlier that failure in compression is initiated and driven by the shear component of the traction vector acting on the fracture plane. The material model is reduced to developing a physical constitutive law for the traction vector, which for example couples damage and friction.

\section{Coupling damage and friction: one dimensional shear response}

Assume a portion of material subjected to a one dimensional, constant, compressive normal load $\sigma$ as well as a monotically increasing shear deformation $\gamma$, the latter resulting in a shear stress $\tau$. Initially, the material behaves linear elastically, up to a given stress at which damage initiates. In tension, the area damaged would not carry any stress, but, in compression, contact takes place between microcrack faces and a friction force is created. Ragueneau et al. ${ }^{9}$ proposed a model for coupling the effect of damage and friction. In a one dimensional form, the model is written as

$$
\tau=(1-d) G \gamma+d \cdot \tau^{\text {friction }}
$$

where $d$ is the damage variable, $G$ the shear modulus and the friction term is found through a Coulomb law, that is to say

$$
\tau^{\text {friction }}=G\left(\gamma-\gamma_{s}\right) \text { if sliding does not takes place }
$$

$$
\tau^{\text {friction }}=-\mu \sigma \quad \text { if sliding takes place }
$$

where $\gamma_{s}$ is a sliding strain, $\mu$ is a coefficient of friction and the contact stiffness is assumed to be equal to the shear modulus $G$ for simplicity. To illustrate equation (1), an example is created with a damage variable, $d$, evolving so that a linear softening of the stress is obtained, if all other effects are neglected. Figure 3 shows the shear responses for different levels of compressive normal stresses, as well as the evolution of the damage variable, which is identical for all pressure values. Three regions are observed in Figure 3, marked A, B and C. Region A corresponds to the linear elastic region, i.e. where the damage variable $d=0$ in equation (1). Region $\mathrm{B}$ corresponds to a 


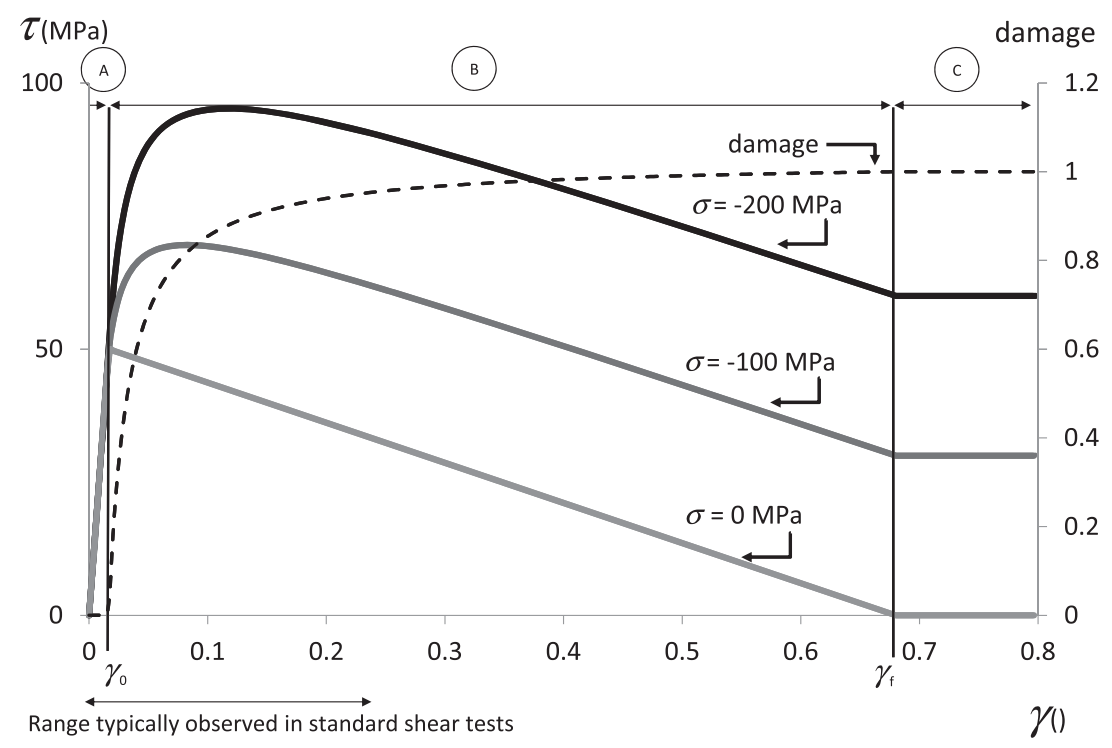

Figure 3. Illustration in one dimension of the shear response for a damage model couple to friction.

range where both friction and damage are acting on the response and counter balance each other, giving rise to the nonlinearity observed. No plasticity or other nonlinear material features are introduced. Finally, region C corresponds to $d=1$ in equation (1), i.e. only the friction term is left. Each curve in Figure 3 was generated using a constant compressive stress, which gives therefore a constant friction term according to equation (3). However, this is not always the case in an arbitrary 3D loading.

It is worth noting how the increase in compressive normal stress gives an increase of the maximum shear stress allowable. This is a result of friction alone, as the fracture toughness, controlling the evolution of damage, is kept constant for the three curves.

\section{General formulation}

This section describes the mathematical aspects of the model described before, i.e. elastic response, damage initiation, split of the stress in damage and friction part, evolution of damage and evolution of friction.

\section{Elastic response and trial stress}

The material is defined as transversely isotropic with a linear elastic behaviour, so that the trial stress, $\tilde{\boldsymbol{\sigma}}$, is related to the strain through the stiffness matrix $C$

$$
\tilde{\boldsymbol{\sigma}}=\boldsymbol{C}: \boldsymbol{\varepsilon}
$$

The trial stress is rotated to a set of potential fracture planes oriented at an angle $\alpha$, as shown in Figure 2, through $\boldsymbol{T}$, the transformation matrix from the $(1,2,3)$ coordinate system to the $(\mathrm{N}, \mathrm{L}, \mathrm{T})$ coordinate system

$$
\begin{gathered}
\tilde{\boldsymbol{\sigma}}^{\mathrm{NLT}}=\boldsymbol{T}: \tilde{\boldsymbol{\sigma}} \\
\boldsymbol{\varepsilon}^{\mathrm{NLT}}=\boldsymbol{T}: \boldsymbol{\varepsilon}
\end{gathered}
$$

where $\mathrm{N}$ refers to the normal direction to the fracture plane, $\mathrm{T}$ is the transverse direction to the fibre and $\mathrm{L}$ is the longitudinal direction to the fibres.

The traction vector is defined as

$$
\tilde{\boldsymbol{\sigma}}^{\mathrm{NLT}} \cdot \boldsymbol{n}=\left(\begin{array}{lll}
\tilde{\sigma}_{\mathrm{N}} & \tilde{\tau}_{\mathrm{L}} & \tilde{\tau}_{\mathrm{T}}
\end{array}\right)^{T}
$$

and the corresponding strain is

$$
\boldsymbol{\varepsilon}^{\mathrm{NLT}} \cdot \boldsymbol{n}=\left(\begin{array}{lll}
\varepsilon_{\mathrm{N}} & \gamma_{\mathrm{L}} & \gamma_{\mathrm{T}}
\end{array}\right)^{T}
$$

where $\boldsymbol{n}=\left(\begin{array}{lll}1 & 0 & 0\end{array}\right)^{T}$ in the $(\mathrm{N}, \mathrm{L}, \mathrm{T})$ coordinate system.

\section{Damage initiation}

The traction vector defined in equation (7) is used to calculate a damage initiation criterion, on multiple potential fracture planes, defined at a given time $t$ of an analysis as

$$
f(t)=\max _{\substack{\mathrm{t}^{\prime} \leq t \\ \alpha \in[0, \pi]}}\left\{\left(\frac{\tilde{\tau}_{\mathrm{L}}}{S_{\mathrm{L}}}\right)^{2}+\left(\frac{\tilde{\tau}_{\mathrm{T}}}{S_{\mathrm{T}}}\right)^{2}\right\}
$$


where $S_{\mathrm{L}}$ and $S_{\mathrm{T}}$ are the longitudinal and transverse shear strengths, respectively. The strength here refers to the initiation of damage, i.e. the start of region $\mathrm{B}$ in Figure 3, and not to the maximum shear stress allowable. The final value of the fracture plane angle $(\alpha)$ is chosen as the one that maximises $f$.

\section{Thermodynamic definition and split of the stress tensor}

Once damage is initiated and the fracture plane is found, the material response is calculated using the constitutive law acting on the fracture plane. For this constitutive law, it is assumed that part of the energy is dissipated by damage formation and the rest by friction during sliding - giving rise to permanent strain during unloading. Permanent strains are only induced by sliding and therefore the total strain is used in the calculation of the damaged stress. This formulation is similar to the one proposed in a continuum framework by Ragueneau et al., ${ }^{9}$ but is here applied to the traction vector acting on the fracture plane and its associated strain vector. We follow the work by Ragueneau et al. ${ }^{9}$ to define the state potential for such a system

$$
\begin{aligned}
\rho \psi= & \frac{1}{2}(1-d) \cdot \boldsymbol{\varepsilon}^{\mathrm{NLT}} \boldsymbol{K} \boldsymbol{\varepsilon}^{\mathrm{NLT}}+\frac{1}{2} d \cdot\left(\boldsymbol{\varepsilon}^{\mathrm{NLT}}-\boldsymbol{\varepsilon}_{\boldsymbol{s}}\right) \\
& \boldsymbol{K}\left(\boldsymbol{\varepsilon}^{\mathrm{NLT}}-\boldsymbol{\varepsilon}_{\boldsymbol{s}}\right)
\end{aligned}
$$

where $\rho$ is the density of the material, $\psi$ the state potential, $d$ a damage variable, $\varepsilon_{s}=\left(\begin{array}{lll}0 & \gamma_{s \mathrm{~L}} & \gamma_{s \mathrm{~T}}\end{array}\right)^{T}$ the sliding strain vector and $\boldsymbol{K}$ the stiffness matrix between the traction vector and its associated strain vector

$$
\boldsymbol{K}=\left[\begin{array}{ccc}
E_{N} & 0 & 0 \\
0 & G_{L} & 0 \\
0 & 0 & G_{T}
\end{array}\right]
$$

The stiffness matrix in equation (11) is calculated from the trial traction vector as

$$
E_{N}=\tilde{\sigma}_{N} / \varepsilon_{N}, G_{L}=\tilde{\tau}_{L} / \gamma_{L} \text { and } G_{T}=\tilde{\tau}_{T} / \gamma_{T}
$$

The energy dissipated by the material under loading (only mechanical loading is considered) has to be positive, and the Clausius-Duhem condition must be fulfilled

$$
\boldsymbol{\sigma}^{\mathrm{NLT}} \dot{\boldsymbol{\varepsilon}}^{\mathrm{NLT}}-\rho \dot{\psi} \geq 0
$$

where $\dot{\psi}=\frac{\partial \psi}{\partial \varepsilon^{N L T}} \dot{\boldsymbol{\varepsilon}}^{\mathrm{NLT}}+\frac{\partial \psi}{\partial \boldsymbol{\varepsilon}_{s}} \dot{\boldsymbol{\varepsilon}}_{S}+\frac{\partial \psi}{\partial d} \dot{d}$ and gives the following

$$
\left(\boldsymbol{\sigma}^{\mathrm{NLT}}-\rho \frac{\partial \psi}{\partial \boldsymbol{\varepsilon}^{N L T}}\right) \dot{\boldsymbol{\varepsilon}}^{\mathrm{NLT}}-\rho \frac{\partial \psi}{\partial \boldsymbol{\varepsilon}_{s}} \dot{\boldsymbol{\varepsilon}}_{s}-\rho \frac{\partial \psi}{\partial d} \dot{d} \geq 0
$$

From equation (13), the following state laws are defined

$$
\begin{aligned}
& \boldsymbol{\sigma}^{\mathrm{NLT}}=\rho \frac{\partial \psi}{\partial \boldsymbol{\varepsilon}^{\mathrm{NLT}}}=(1-d) \boldsymbol{K} \boldsymbol{\varepsilon}^{\mathrm{NLT}}+d \cdot \boldsymbol{K}\left(\boldsymbol{\varepsilon}^{\mathrm{NLT}}-\boldsymbol{\varepsilon}_{s}\right) \\
& \boldsymbol{\sigma}_{s}=-\rho \frac{\partial \psi}{\partial \boldsymbol{\varepsilon}_{s}}=d \boldsymbol{K}\left(\boldsymbol{\varepsilon}^{\mathrm{NLT}}-\boldsymbol{\varepsilon}_{s}\right)=d \cdot \boldsymbol{\sigma}^{\text {friction }} \quad \text { (b) } \\
& G=\rho \frac{\partial \psi}{\partial d}=-\frac{\boldsymbol{K} \boldsymbol{\varepsilon}_{s}}{2}\left(2 \boldsymbol{\varepsilon}^{\mathrm{NLT}}-\boldsymbol{\varepsilon}_{s}\right) \quad \text { (c) }
\end{aligned}
$$

where $\sigma_{\mathrm{s}}$ is the sliding stress.

The term $-\rho \frac{\partial \psi}{\partial \boldsymbol{\varepsilon}_{s}} \dot{\boldsymbol{\varepsilon}}_{s}=\boldsymbol{\sigma}_{\boldsymbol{s}} \dot{\boldsymbol{\varepsilon}}_{s}$ in equation (13) is positive because of the Kuhn-Tucker conditions imposed on sliding, as detailed in the Sliding criterion section. It is therefore sufficient to ensure that the last term in equation (13) is positive. The evolution of damage is irreversible ensuring $\dot{\boldsymbol{d}} \geq 0$ so that only one condition remains on the sliding strains, namely that $\boldsymbol{\varepsilon}_{\boldsymbol{s}}\left(2 \boldsymbol{\varepsilon}^{\mathrm{NLT}}-\boldsymbol{\varepsilon}_{\boldsymbol{s}}\right) \geq 0$. Under the assumption that damage is driven by the shear component, equation (14a) can be rewritten for $\tilde{\sigma}_{N} \leq 0$ as

$$
\begin{aligned}
\boldsymbol{\sigma}^{\mathrm{NLT}} & =(1-d) \tilde{\boldsymbol{\sigma}}^{\mathrm{NLT}}+d \cdot \boldsymbol{\sigma}^{\text {friction }} \\
& =\left(\begin{array}{c}
\tilde{\sigma}_{N} \\
(1-d) \tilde{\tau}_{L} \\
(1-d) \tilde{\tau}_{T}
\end{array}\right)+\left(\begin{array}{c}
0 \\
d \cdot \tau_{L}^{\text {friction }} \\
d \cdot \tau_{T}^{\text {friction }}
\end{array}\right)
\end{aligned}
$$

\section{Damage evolution law}

Once damage initiation is predicted, i.e. $f=1$, the damage variable, $d$, is calculated as function of the driving strain $\gamma$

$$
d=1-\frac{\gamma_{0}}{\gamma}\left(\frac{\gamma_{f}-\gamma}{\gamma_{f}-\gamma_{0}}\right)
$$

Equation (16) corresponds to a linearly decreasing softening traction separation law as in Pinho et al. ${ }^{1}$ The driving strain is defined as follows

$$
\gamma=\sqrt{\left(\gamma_{L}\right)^{2}+\left(\gamma_{T}\right)^{2}}
$$

where $\gamma_{L}$ and $\gamma_{T}$ are the shear strains in the longitudinal and transverse directions, respectively. The shear strain at damage initiation in equation (16) is defined as

$$
\gamma_{0}=\sqrt{\left(\gamma_{L, 0}\right)^{2}+\left(\gamma_{T, 0}\right)^{2}}
$$

where $\gamma_{L, 0}$ and $\gamma_{T, 0}$ are the shear strains at damage initiation in the transverse and longitudinal direction, respectively, see Figure 2. 
The strain $\gamma_{f}$ is dependent of the toughness of the material, $G_{\mathrm{c}}$, and is calculated as

$$
\gamma_{f}=2\left(G_{c} / L_{c}\right) / \tau_{0}
$$

where $\tau_{0}$ is the shear stress at damage initiation and $L_{c}$ is the characteristic length of a finite element (FE). ${ }^{1}$

To account for mixed mode, the fracture toughness in equation (19) is defined as in Pinho et al. ${ }^{1}$

$$
G_{C}=G_{I I C}\left(\frac{\gamma_{L, 0}}{\gamma}\right)^{2}+G_{I I I C}\left(\frac{\gamma_{T, 0}}{\gamma}\right)^{2}
$$

The mode I component is removed here as only compressive loading is assumed. In the rest of this paper, it is assumed that $G_{I I C}=G_{I I I C}$.

\section{Sliding criterion}

The frictional part of the shear stress is based on a Coulomb sliding criterion.

If $\tilde{\sigma}_{N} \leq 0$, then

$$
\phi=\left\|\boldsymbol{\sigma}^{\text {friction }}\right\|+\mu\left(\tilde{\sigma}_{N}-p_{0}\right)
$$

where $\phi$ is the function defining the sliding criterion and $p_{0}$ is introduced to account for an apparent internal pressure, e.g. residual stresses built up during manufacturing. Both $p_{0}$ and the coefficient of friction $\mu$ can account for anisotropic behaviour ${ }^{1}$

$$
\begin{aligned}
& \mu=\sqrt{\left(\mu_{T} \cos \theta\right)^{2}+\left(\mu_{L} \sin \theta\right)^{2}} \\
& p_{0}=\sqrt{\left(p_{0 T} \cos \theta\right)^{2}+\left(p_{0 L} \sin \theta\right)^{2}} \\
& \text { with } \theta=\arctan \left(\tau_{L} / \tau_{T}\right) \quad \text { (c) }
\end{aligned}
$$

A sliding multiplier giving the increment in sliding strain and used to calculate the friction stress in equation $14(\mathrm{~b})$ is defined as

$$
\dot{\boldsymbol{\varepsilon}}_{S}=\dot{\lambda} \frac{\partial \phi}{\partial \boldsymbol{\sigma}^{\text {friction }}}=\dot{\lambda}
$$

Finally, Khun-Tucker conditions are defined to determine if sliding occurs or not

$$
\dot{\lambda} \geq 0, \quad \phi \leq 0, \quad \dot{\lambda} \phi=0
$$

\section{Finite element implementation}

\section{Stress integration and computational procedure}

The integration of the stress is performed incrementally over a sequence of time steps $\left[t_{n}, t_{n+1}\right]$ with a strain driven process, i.e. the value of the new strain at time $t_{n+1}$ is given as $\varepsilon_{n+1}=\varepsilon_{n}+\varepsilon_{n+1}$. The method of additive operator (stress) split proposed in Simo et al. ${ }^{11}$ is used here; that is to say that the damage component and the frictional component of the stress are integrated in parallel. Each component of the stress uses a return mapping corrector algorithm, i.e. an elastic trial stress (also called predictor) is first calculated to assess if damage or friction is activated. If one condition is fulfilled, then the appropriate corrector is calculated and applied to the stress.

The following algorithm is used:

A1. Initial conditions

$$
\boldsymbol{\varepsilon}_{n}, \Delta \boldsymbol{\varepsilon}_{n+1}, \boldsymbol{\sigma}_{n}, f_{n}, d_{n}, \boldsymbol{\sigma}_{s, n}, \boldsymbol{\varepsilon}_{s, n} .
$$

A2. Update strain and elastic trial stress/predictor for the damage stress

$$
\begin{gathered}
\boldsymbol{\varepsilon}_{n+1}=\boldsymbol{\varepsilon}_{n}+\Delta \boldsymbol{\varepsilon}_{n+1} \\
\tilde{\boldsymbol{\sigma}}_{\mathrm{n}+1}=\boldsymbol{C}: \boldsymbol{\varepsilon}_{n+1}
\end{gathered}
$$

A3. Failure index and check for damage initiation

$$
\begin{gathered}
\tilde{\boldsymbol{\sigma}}_{\mathrm{n}+1}^{\mathrm{NLT}} \cdot \boldsymbol{n}=\left(\boldsymbol{T}: \tilde{\boldsymbol{\sigma}}_{\mathrm{n}+1}\right) \cdot \boldsymbol{n} \\
\tilde{f}_{\mathrm{n}+1}=\left(\frac{\tilde{\tau}_{\mathrm{L}, \mathrm{n}+1}}{S_{L}}\right)^{2}+\left(\frac{\tilde{\tau}_{\mathrm{T}, \mathrm{n}+1}}{S_{T}}\right)^{2} \\
f_{\mathrm{n}+1}= \begin{cases}f_{\mathrm{n}} & \text { if } f_{\mathrm{n}+1} \leq f_{\mathrm{n}} \\
\tilde{f}_{\mathrm{n}+1} & \text { if } f_{\mathrm{n}+1}>f_{\mathrm{n}} \\
1 & \text { if } f_{\mathrm{n}+1} \geq 1\end{cases}
\end{gathered}
$$

Repeat A3 for $\alpha \in\left[0^{\circ} ; 180^{\circ}[\right.$ fixed

If $f_{\mathrm{n}+1}<1$, then $\boldsymbol{\sigma}_{\mathrm{n}+1}=\tilde{\boldsymbol{\sigma}}_{\mathrm{n}+1}$, otherwise $\alpha$ is

A4. Damage variable and damage corrector

$$
\begin{aligned}
& \tilde{d}_{\mathrm{n}+1}=1-\frac{\gamma_{0}}{\gamma_{n+1}}\left(\frac{\gamma_{f}-\gamma_{n+1}}{\gamma_{f}-\gamma_{0}}\right) \\
& d_{\mathrm{n}+1}= \begin{cases}d_{\mathrm{n}} & \text { if } d_{\mathrm{n}+1} \leq d_{\mathrm{n}} \\
\tilde{d}_{\mathrm{n}+1} & \text { if } d_{\mathrm{n}+1}>d_{\mathrm{n}} \\
1 & \text { if } d_{\mathrm{n}+1} \geq 1\end{cases} \\
& \boldsymbol{\sigma}_{\mathrm{n}+1}^{\mathrm{NLT}}=\left(1-d_{\mathrm{n}+1}\right) \boldsymbol{K}_{\mathrm{n}+1}^{\mathrm{NLT}}=\left(1-d_{\mathrm{n}+1}\right) \tilde{\boldsymbol{\sigma}}_{\mathrm{n}+1}^{\mathrm{NLT}}
\end{aligned}
$$

A5. Elastic trial stress/predictor for the frictional stress

$$
\tilde{\boldsymbol{\sigma}}_{n+1}^{\text {friction }}=\boldsymbol{K}\left(\boldsymbol{\varepsilon}_{\mathrm{n}+1}^{\mathrm{NLT}}-\boldsymbol{\varepsilon}_{s, n}\right)
$$


A6. Check sliding

$$
\tilde{\phi}_{n+1}=\left\|\tilde{\boldsymbol{\sigma}}_{n+1}^{\text {friction }}\right\|+\mu\left(\tilde{\sigma}_{N, n+1}-p_{0}\right)
$$

If $\tilde{\phi}_{n+1}<0$ then sticking is taking place, i.e. $\boldsymbol{\varepsilon}_{s, n+1}=\boldsymbol{\varepsilon}_{s, n}$, and go to A8, otherwise

A7. Frictional stress corrector

$$
\begin{aligned}
\dot{\lambda}_{n+1} & =\left(\begin{array}{c}
\tilde{\phi}_{n+1} / G_{L} \\
\tilde{\phi}_{n+1} / G_{T}
\end{array}\right) \\
\boldsymbol{\varepsilon}_{S, n+1} & =\boldsymbol{\varepsilon}_{S, n}+\dot{\boldsymbol{\lambda}}_{n+1} \Delta t \\
\boldsymbol{\sigma}_{n+1}^{\text {friction }} & =\left(\begin{array}{c}
\tau_{L, n+1}^{\text {friction }} \\
\tau_{T, n+1}^{\text {friction }}
\end{array}\right)=\left(\begin{array}{c}
-\mu\left(\tilde{\sigma}_{N, n+1}-p_{0}\right) \cdot \sin \theta \\
-\mu\left(\tilde{\sigma}_{N, n+1}-p_{0}\right) \cdot \cos \theta
\end{array}\right) \\
\boldsymbol{\sigma}_{\mathrm{n}+1}^{\mathrm{NLT}} & =\boldsymbol{\sigma}_{\mathrm{n}+1}^{\mathrm{NLT}}+d_{\mathrm{n}+1} \boldsymbol{\sigma}_{n+1}^{\text {friction }}
\end{aligned}
$$

A8. Return stress to material axis.

\section{Characteristic length and smeared model}

Fixed crack models have been successful in modelling damage in concrete and are often used in combination with a smeared crack formulation. ${ }^{1}$ In such a formulation, the area under the stress strain curve, corresponding to a fracture energy per unit volume, is corrected by a characteristic length (typically a finite element) to ensure that the fracture energy per unit area is dissipated independently of the element size.

In the present contribution, only cubic elements of edge length $L$ are used, and the definition given by Bažant and $\mathrm{Oh}^{12}$ for the characteristic length, associated with a fracture plane oriented at an angle $\alpha$, is adopted here

$$
L_{c}=\frac{L^{2}}{\cos (\alpha)}
$$

Smeared softening models suffer from stress locking because of the continuity in displacement of the finite element method. ${ }^{13}$ This results in spurious cracking, if damage has initiated in that element, and spurious stiffening otherwise. Jirásek and Zimmermann ${ }^{14}$ proposed a combination of the fixed crack model with an isotropic scalar damage model to eliminate this difficulty. Such an approach is followed in the present paper. When the shear strain reaches the value of 1 $(100 \%)$, all stress components are smoothly decreased to zero.

\section{Model parameters identification}

The model developed in the General formulation section requires that both the longitudinal and transverse directions are characterised to obtain the strength, damage and friction properties required by the model and listed in Table 1. These directions correspond to the in-plane $\left(\tau_{12}\right)$ and the through-thickness shear $\left(\tau_{23}\right)$ for a unidirectional composite when $\alpha=0^{\circ}$, as shown Figure 4.

Besides elastic properties, the parameters required for the model can be subdivided in three categories: (i) strength properties, (ii) damage properties and (iii) friction properties. In the experimental and numerical studies presented below, a carbon epoxy system T700/MTM57 has been used and the properties for this material are summarised in Table 1. The elastic and strength properties are obtained from the material supplier. The characterisation of the longitudinal shear properties is presented below.

\begin{tabular}{|c|c|c|c|c|}
\hline \multicolumn{5}{|c|}{ Elastic properties } \\
\hline \multicolumn{2}{|l|}{ Modulus } & \multicolumn{3}{|c|}{ Poisson's coefficient } \\
\hline Longitudinal & Transverse & Longitudinal shear & In-plane & Out of plane \\
\hline$E_{11}(\mathrm{GPa})$ & $E_{22}(\mathrm{GPa})$ & $G_{12}(\mathrm{GPa})$ & $v_{12}()$ & $v_{23}()$ \\
\hline 128 & 7.9 & 3 & 0.3 & 0.4 \\
\hline \multicolumn{3}{|c|}{ Strength properties } & \multicolumn{2}{|l|}{ Damage properties } \\
\hline Longitudinal shear & Transverse shear & & Mode II Fracture toughness & \\
\hline$S_{L}(\mathrm{MPa})$ & $\mathrm{S}_{T}(\mathrm{MPa})$ & & $G_{l l c}\left(\mathrm{~kJ} / \mathrm{m}^{2}\right)$ & \\
\hline 50 & 60 & & 2 & \\
\hline \multicolumn{5}{|c|}{ Friction properties } \\
\hline Longitudinal & Transverse & Longitudinal & Transverse & \\
\hline POL $(\mathrm{MPa})$ & Рот (MPa) & $\mu_{L}()$ & $\mu_{T}()$ & \\
\hline 75 & 30 & 0.3 & 0.3 & \\
\hline
\end{tabular}
Data for the transverse shear response are, however, not available; therefore, $S_{\mathrm{T}}$ is calculated using equation (26) ${ }^{15}$ with $Y_{C}=160 \mathrm{MPa},{ }^{16} \mu_{T}$ is assumed equal to $\mu_{L}$ and $p_{0 T}$ is found by matching the

Table I. Material properties for T700/MTM57. 


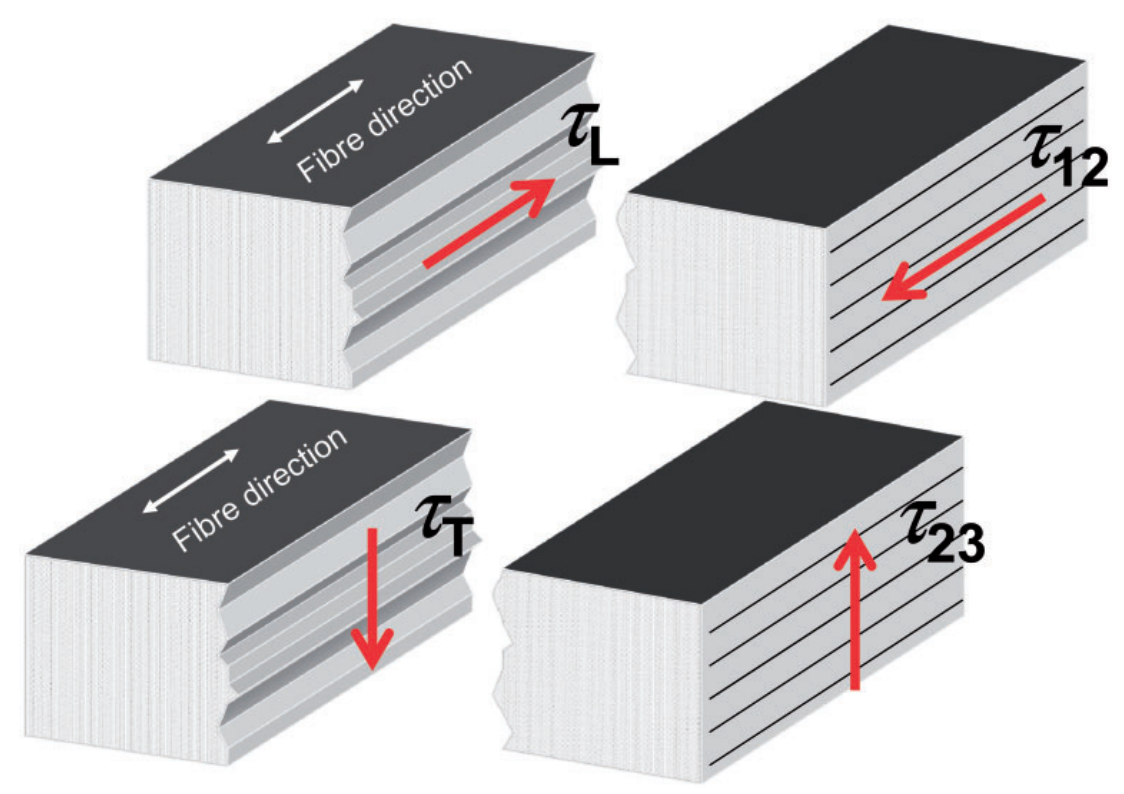

Figure 4. Illustration of relation between in-plane and through-thickness stresses to longitudinal and transverse shear stresses on the fracture plane for $\alpha=0^{\circ}$.

(a)

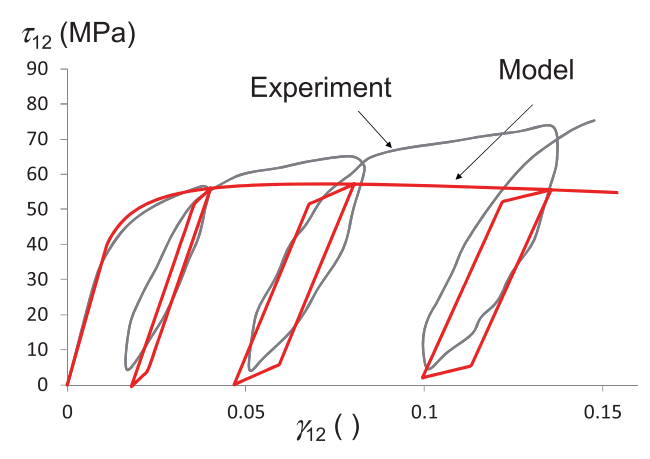

(b)

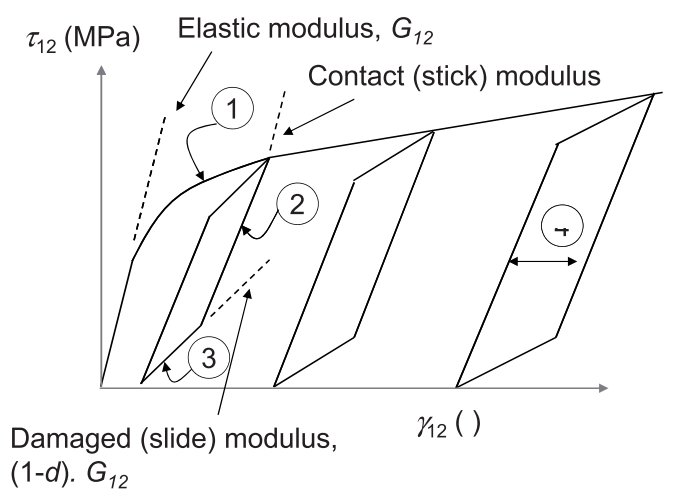

Figure 5. (a) Shear response for MTM57/T700, experimental from Totry et al. ${ }^{17}$ on MTM57/E-glass; (b) schematic of the cyclic response.

response of transverse compression tests presented in Casado. $^{16}$

$$
S_{T}=\frac{Y_{C}}{2 \mu_{T}\left(1+\sqrt{1+1 / \mu_{T}^{2}}\right)}
$$

Figure 5(a) shows the identification of the shear response with experimental longitudinal shear tests from Totry et al. ${ }^{17}$ (note that, in Totry et al., ${ }^{17}$ the loading/unloading tests are performed on an E-Glass system, but it is also shown there that this system and T700/
MTM57 have the same behaviour for strain under $10 \%)$. The experimental response was obtained in Totry et al. ${ }^{17}$ using a Iosipescu testing rig with a double notched specimen with a $[0 / 90]_{4 S}$ layup. The shear stressstrain response is measured using the loading cell of the testing equipment and digital image correlation for the strain. It is assumed here that the measured response is representative of the material behaviour in shear. Therefore, the identification is performed by using a single finite element loaded in pure shear, and not by modelling the whole testing rig. The schematic in Figure 5(b) describes the different stages during a cyclic loading, and it should be noted that Figure 5(a) corresponds to the range $(0-0.15)$ shown in Figure 3. 
After damage initiation, the non-linear response is a result of the decreasing elastic traction due to damage growth and an increasing frictional shear component, labelled stage 1 in Figure 5(b). The magnitude of the non-linear response is a function of the instantaneous damage variable $d$ and of the frictional force, i.e. the product $\mu_{L} p_{0 L}$.

If unloading takes place after damage initiation, then stick behaviour with contact stiffness $G_{12}$ is first observed, labelled stage 2 in Figure 5(b), then this is followed by elastic slip with a stiffness $(1-d) G_{12}$, labelled stage 3 in Figure 5(b).

The evolution of the damage variable $d$ (and therefore the fracture energy $G_{I I C}$ ) as a function of strain is calculated from the stiffness in stage 3 at several unloading cycles. The constant internal pressure $p_{0 L}$ is identified from stage 1 by matching the non-linear response and the longitudinal coefficient of friction $\mu_{L}$ is found from matching the width of hysteresis loops (stage 4) at several strain levels.

\section{Combined longitudinal shear and transverse loading}

The model is studied for several combinations of inplane shear loading and transverse compressive loading $\left(\tau_{12}, \sigma_{22}\right)$. The normalised failure envelopes of three different material systems (E-Glass/LY556, ${ }^{18}$ T800/ $3900-2^{19}$ and AS4 $/ 55 \mathrm{~A}^{20}$ ) are given in Figure 6. The mechanical properties for these materials are given in Table 2. ${ }^{18,21}$ The coefficients of friction are chosen so that $\mu_{T}=\mu_{L}=0.3$; the apparent internal pressures are shown in Table 1, and the transverse shear strength values are calculated using equation (26). The normalised envelopes predicted by the model for the three materials are very similar and only the envelope for the T800/3900-2 system is plotted.

Experimentally, the three material systems show similar trends in Figure 6, with an increase of the maximum shear stress allowable with moderate compressive stresses. This trend is captured by the model and it should be noted that the dependency of the shear strength with the transverse stress is only a result of friction (as shown in Figure 3), as the initiation criterion, equation (9), does not depend on the normal stress. The difference between damage onset predicted by equation (9) and the maximum stress carried is also shown in Figure 6. It can be seen that the ability of carrying stress by friction is quite significant. The fracture plane angles are also reported in Figure 6. Angles are searched for with a $15^{\circ}$ increment in the model, which was found to be the best compromise between accuracy and computational efficiency. The maximum angle of $45^{\circ}$ reported in Figure

Table 2. Material properties used in Figure 6.

\begin{tabular}{lcllllr}
\hline & $E_{11}$ & $E_{22}$ & $G_{12}$ & \multicolumn{1}{l}{$S_{\mathrm{L}}$} & $Y_{\mathrm{T}}$ & \multicolumn{1}{c}{$Y_{\mathrm{C}}$} \\
\hline & $(\mathrm{GPa})$ & $(\mathrm{GPa})$ & $(\mathrm{GPa})$ & $(\mathrm{MPa})$ & $(\mathrm{MPa})$ & $(\mathrm{MPa})$ \\
E-Glass/LY556 & 53.4 & 17.7 & 5.8 & 66.5 & 37.5 & 130.3 \\
AS4/55A & 126 & 11 & 6.6 & 51.3 & 27 & 91.8 \\
T800/3900-2 & 155 & 8.5 & 5.5 & 100.9 & 48.8 & 201.7 \\
\hline
\end{tabular}

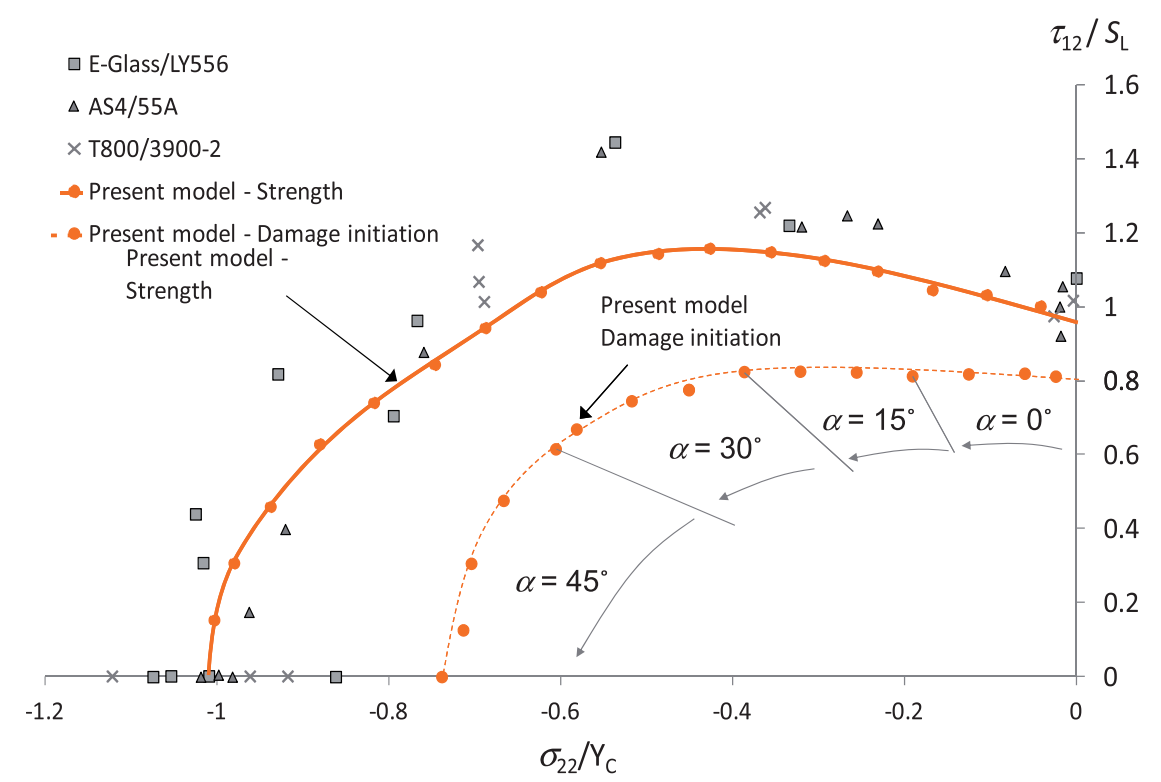

Figure 6. Combined longitudinal shear and transverse compression failure envelopes E-Glass/LY556, ${ }^{18} \mathrm{~T} 800 / 3900-2^{19}$ and AS4 $/ 55 \mathrm{~A}^{20}$. 
6 is lower than values of $53^{\circ}$ typically reported in the literature, ${ }^{1}$ as it corresponds here to the angle at microcracks initiation and not the angle of the final macrocracks.

\section{Application to crushing}

To validate the model, a simple experimental investigation on the crushing of a wedge specimen is designed. Typical crushing experiments are performed on multi-directional tubes ${ }^{22}$ and corrugated specimens. ${ }^{23,24}$ These designs, which are aimed at studying practical stacking sequences and prevent buckling during crushing, are however difficult to use for model validation. The sequence of events during failure is intricate with damage in the plies with different orientation interacting. The circular or corrugated shapes are an added difficulty which may overshadow issues related to material modelling. The experiments presented here are an attempt to trigger crushing on a simplified geometry and layup, by using short unidirectional specimens with a wedge as initiator. The specimens have geometries as simple as possible so that any uncertainty regarding the loading conditions or geometrical features can be removed. Furthermore, a unidirectional layup is chosen to isolate the damage mechanisms at a single ply level.

\section{Experiments}

The specimens were made of a carbon-epoxy system (T700/MTM57), and laid up as unidirectional [90 $]_{8 \mathrm{~S}}$ laminate to obtain a cured thickness of $2.5 \mathrm{~mm}$. The specimens were manufactured and cured in an autoclave according to the manufacturer's recommendation. Five specimens were then cut to the dimensions shown in Figure 7(a) using a band saw, and one end was chamfered at an angle $\omega$, equal to $10^{\circ}$.

The specimens were then clamped (hand tightened) between two metal blocks over a length of $16 \mathrm{~mm}$, leaving $4 \mathrm{~mm}$ for crushing, see Figure 7(b). Support and specimens were then placed between two loading plates, and crushing took place at a quasi-static rate

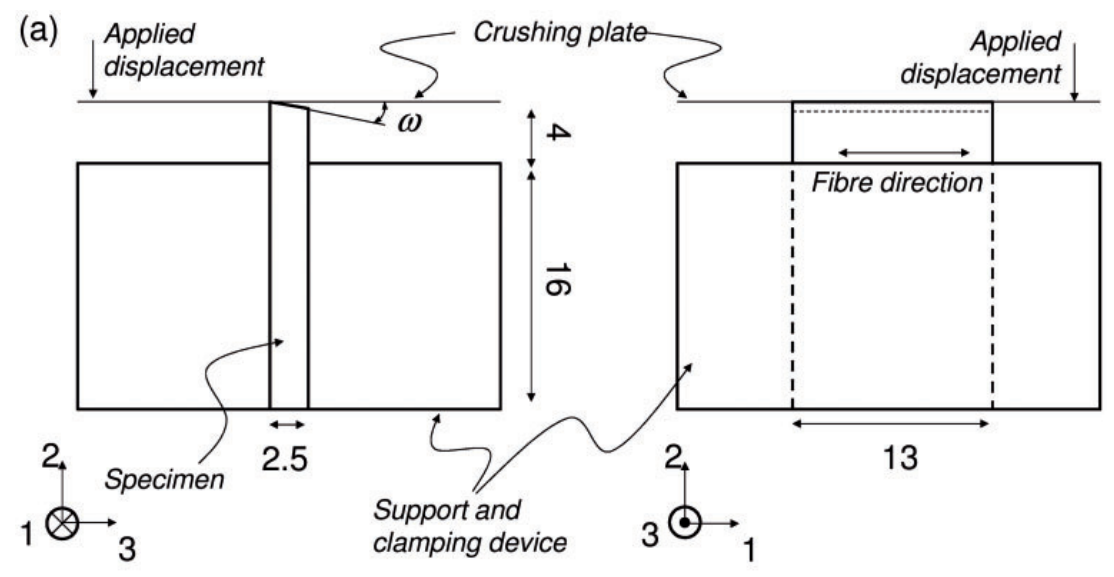

(b)

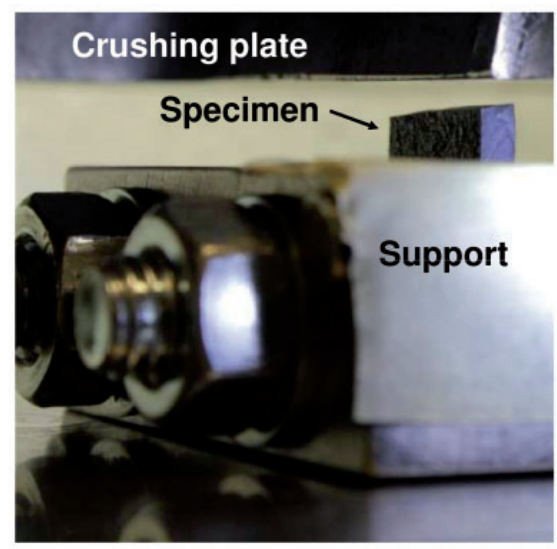

Figure 7. (a) Experimental setup and (b) schematic of the setup with dimensions in $\mathrm{mm}$. 
of $0.5 \mathrm{~mm} / \mathrm{min}$. The thin side of the specimen was observed with an in situ microscope.

\section{Finite element model}

A 3D finite element model of the experimental setup has been developed, see Figure 8. The specimen is modelled using quad elements with reduced integration (C3D8R in Abaqus). The mesh size is $0.1 \mathrm{~mm}$ to capture the geometrical features (including the wedge) with high fidelity. The support and crushing plates are represented as rigid bodies and the contact between the specimen and plate is accounted for with a general contact including friction (with a coefficient of friction of $0.16)^{25}$. The failure process is similar throughout the specimen width (in the 1-direction), therefore only a cross section of the specimen is modelled using one row of elements. The displacements in the 1-direction on the faces with normals $(1,0,0)$ and $(-1,0,0)$ are constrained so that the faces remain flat, and so that the model represents the material in the centre of the specimen.

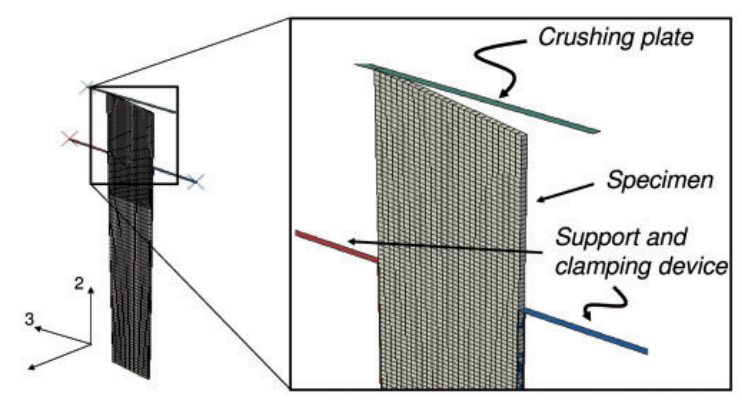

Figure 8. Finite element model with close view on the wedge.
The material model developed in the General formulation section is implemented in a Fortran user subroutine for the commercial finite element package Abaqus/ Explicit. The simulations are run quasi-statically with a low velocity over a long time period. The density of the material is increased by three orders of magnitude in order to increase the time increment. The kinetic energy is monitored during the analysis so that it does not excess $1 \%$ of the strain energy.

\section{Results}

The fracture topologies and failure stresses are consistent for all specimens but large variations in compliance are observed on the load versus displacment curves shown in Figure 9 (note that the exact compliance of the rig was measured separately and substracted from the experimental results presented in Figure 9). The response appears to be initially linear, some nonlinearities then appear before a peak stress is reached, eventually followed by a sharp drop in stress.

In terms of global response, the prediction by the FE model is shown in Figure 9. The slope, up to the maximum load, matches the slope of the stiffest experiments. The model predicts well the level of the peak load. The response of a model where no friction is accounted for is shown as a dashed line in Figure 9. The model without friction is more compliant and the peak stress is approximately $25 \%$ lower than in the experiments.

Figure 10 shows the specimen sidewise during loading; the labels $1-5$ are also shown in Figure 9. The chamfer triggers damage early in the test and is totally crushed at an applied stress of approximately $60 \mathrm{MPa}$, labels 2 and 3. A matrix crack, label 4, forms then

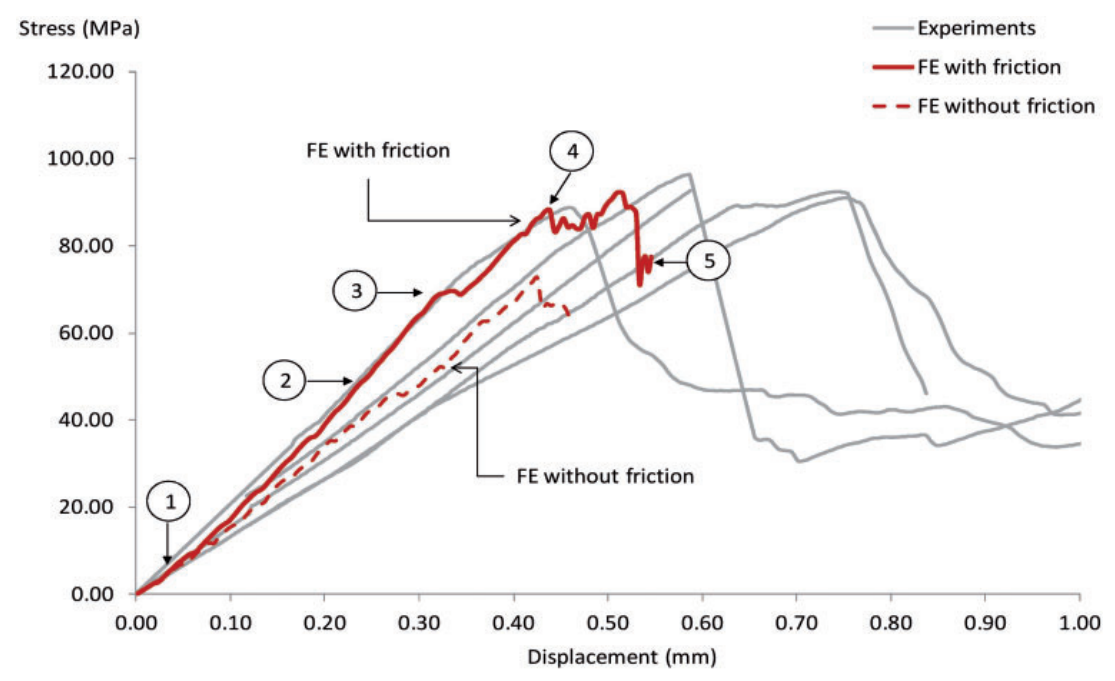

Figure 9. Experimental and numerical response of the $90^{\circ}$ chamfered specimens. 


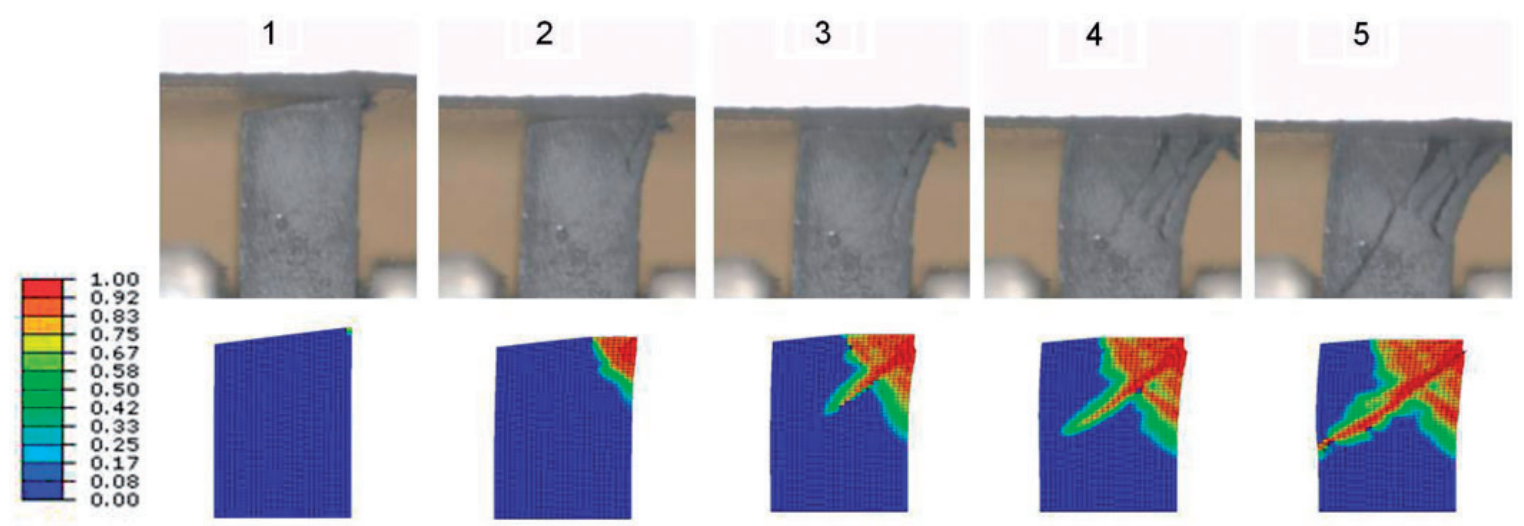

Figure 10. Damage in the specimen during crushing. The scale shows the damage variable $d$.

across the specimen (joining the upper right hand side of the specimen to the opposite side close to the support), leading to a sharp drop in stress, label 5.

The contour plots from the FE models in Figure 10 (the scale shows the damage variable) show a similar damage process and extend at the various stress levels. In particular, right after the drop in stress, the bottom part of the specimen (above the support), characterised by a double sided wedge, is well predicted. The region within the wedge appears to be rather undamaged.

\section{Discussion}

By coupling damage to contact and friction acting on microcracks, several interesting features have been identified. For instance, it is shown in Figure 5(a) that the coupling between damage and friction gives rise to a non-linear response in shear, which is also observed experimentally. The model shows, however, a softening trend while the experimental response exhibits hardening. This difference is attributed to several effects not included in the model, such as possible viscoelastic or non-linear effects of the resin. Also in Figure 5(a), it is interesting to note that the model captures well the hysteresis loops obtained during shear loading and unloading. The hysteresis loops in the model are only due to the frictional behaviour, and the energy enclosed in these loops corresponds to the frictional energy spent during one cycle.

A new view on the role of friction in predicting failure onset in matrix dominated and compressive loading is also provided by Figure 6. Because newly created microcrack faces can carry frictional stresses, the maximum shear stress the material can carry increases with compressive normal stresses, see Figures 3 and 6. This is a known behaviour for composite materials and is typically modelled using stress-based failure criteria of the Mohr-Coulomb type. ${ }^{26}$ The predictions presented here are, however, obtained using a simple quadratic criterion for the shear components for damage onset, and the increase in shear stress is the result of the friction acting on actual microcrack surfaces.

Material properties required for damage models, i.e. fracture energies, are often difficult to measure. ${ }^{6}$ By noting that compressive failure in composite materials is often driven by shear fracture, the characterisation can be simplified to characterising a shear response. The method described in the Model parameters identification section relies on identifying the properties required by loading and unloading of in-plane and through-thickness shear tests. While standard methods are available for the former, more research is required for the latter.

The experiments conducted on the chamfered $90^{\circ}$ specimens show a large scatter in the measured compliances which comes from a series of factors such as the clamping pressure used and the collinearity of the edge of the chamfer. The numerical model predicts a compliance similar to the stiffest specimen, which is often observed for numerical models as, for example, the clamping devices and other parts of the rig are modelled as rigid parts. Nevertheless, these tests are interesting for validating the present model as damage and crushing occur early in the tests, and therefore influence significantly the responses measured. It is clearly seen in Figure 10 that neglecting the friction leads to an under prediction of the failure stress of approximately $25 \%$. The fracture topology during the crushing of the wedge is well predicted; however, it is noted that the main crack does not have the same orientation in the experiments and the model, as it initiates at the top of the specimen and extend to the support in the former, while it initiates under the notch in the latter.

Finally, the formulation of the model can handle large strains, which is desirable to model crushed 
material. However, some limitations are introduced by the use of the standard finite element method as detailed in the Finite element implementation section. The use of discrete methods, such as the extend finite element method (XFEM), ${ }^{27}$ can make it possible to overcome such limitations and for these methods the present model would equally be applicable.

\section{Conclusions}

A material model for unidirectional fibre-reinforced composites coupling damage to the friction acting on the newly created microcracks is developed. As opposed to existing material model in which microcracks remain traction free under compressive load, the model follows a continuum damage mechanics approach which accounts for contact and friction on microcracks. The model is validated against experimental data and it is shown that friction can account for part of the non-linear response and the hysteresis loops typically observed in the shear response of composites. It is also shown using a simple crushing test that the physics behind crushing are well captured, and that neglecting friction results in approximately $25 \%$ under predicted peak loads.

\section{References}

1. Pinho ST, Iannucci L and Robinson P. Physically based failure models and criteria for laminated fibre-reinforced composites with emphasis on fibre kinking. Part II: FE implementation. Compos Part A 2006; 37: 766-777.

2. Gutkin R and Pinho ST. Practical application of failure models to predict the response of composite structures. In: Proceedings of $18^{\text {th }}$ international conference on composite materials (ICCM18), Jeju, 21-26 August 2011.

3. Maimì P, Camanho PP, Mayago JA, et al. A continuum damage model for composite laminates: Part I Constitutive model. Mech Mater 2007; 39: 897-908.

4. Jirásek M and Patzák B. Models for quasibrittle failure: theoretical and computational aspects. In: Proceedings of ECCM-2001, Cracow, Poland, 26-29 June 2001.

5. Iannucci L and Willows ML. An energy based damage mechanics approach to modelling impact onto woven composite materials - Part I: Numerical models. Compos Part A 2006; 37: 2041-2056.

6. Pinho ST, Robinson P and Iannucci L. Fracture toughness of the tensile and compressive fibre failure modes in laminated composites. Compos Sci Technol 2006; 66: 2069-2079.

7. Tvergaard V. Effect of fibre debonding in a whisker-reinforced metal. Mater Sci Eng A-Struct 1990; 125: 203-213.

8. Chaboche JL, Girard R and Schaff A. Numerical analysis of composite systems by using interphase/interface models. Compos Mech 1997; 20: 3-11.

9. Ragueneau F, La Borderie C and Mazars J. Damage model for concrete-like materials coupling cracking and friction, contribution towards structural damping: first uniaxial applications. Mech Cohes Frict Mater 2000; 5: 607-625.

10. Lemaitre $\mathbf{J}$ and Chaboche JL. Aspects phénoménologique de la rupture par endommagement. Journal de Mécanique Appliquée 1978; 2: 317-365.

11. Simo JC and Ju JW. Strain- and stress-based continuum damage models-II. Computational aspects. Int J Solids Struct 1987; 23: 84-869.

12. Bažant $\mathrm{ZP}$ and $\mathrm{Oh} \mathrm{BH}$. Crack band theory for fracture of concrete. Mater Struct 1983; 16: 155-177.

13. Rots JG and Blaauwendraad. Crack models for concrete: Discrete or smeared? Fixed, multi-directional or rotating? Heron 1989; 34: 1-59.

14. Jirásek $\mathrm{M}$ and Zimmermann $\mathrm{T}$. Rotating crack model with transition to scalar damage. J Eng Sci 1998; 124: 277-284.

15. Pinho ST, Iannucci L and Robinson P. Physicallybased failure models and criteria for laminated fibre-reinforced composites with emphasis on fibre kinking: Part I: Development. Compos Part A 2006; 37: 63-73.

16. Casado L. Experimental and computational micromechanical study of fiber-reinforced polymers. $\mathrm{PhD}$ thesis, Universidad Politécnica de Madrid, 2011.

17. Totry E, Gonzalez C and Llorca J. Mechanical behavior of composite materials: experiments and simulations. Anales de Mecánica de la Fractura 2009; 26: 187-192.

18. Soden PD, Hinton MJ and Kaddour AS. Biaxial test results for strength and deformation of a range of e-glass and carbon fibre reinforced composite laminates: Failure exercise benchmark data. Compos Sci Technol 2002; 62: 1489-1514.

19. Swanson SR. A micro-mechanical model for in-situ compression strength of fiber composite laminates. Transact ASME Ser H, J Eng Mater Technol 1992; 114: 8-12.

20. Swanson SR, Messick MJ and Tian Z. Failure of Carbon/Epoxy lamina under combined stress. J Compos Mater 1987; 21: 619-630.

21. Soden PD, Hinton MJ and Kaddour AS. Lamina properties, lay-up configurations and loading conditions for a range of fibre-reinforced composite laminates. Compos Sci Technol 1998; 58: 1011-1022.

22. Hull D. A unified approach to progressive crushing of fibre-reinforced composite tubes. Compos Sci Technol 1991; 40: 377-421.

23. Grauers L, Olsson R and Gutkin R. Energy absorption and damage mechanisms in progressive crushing of corrugated NCF laminates: Fractographic analysis. Compos Struct 2014; 110: 110-117.

24. Feraboli P. Development of a corrugated test specimen for composite materials energy absorption. $J$ Compos Mater 2008; 42: 229-256.

25. Stocchi C, Robinson P and Pinho ST. A detailed finite element investigation of composite bolted joints with countersunk fasteners. Compos Part A 2013; 52: 143-150.

26. Puck A and Schürmann. Failure analysis of FRP laminates by means of physically based phenomenological models. Compos Sci Technol 1998; 58: 1045-1067.

27. Huynh DBP and Belytschko T. The extended finite element method for fracture in composite materials. Int $J$ Numer Meth Eng 2009; 77: 214-239. 